\title{
Trypanosomose animale chez les bovins \\ dans la zone Sud-soudanienne \\ du Burkina Faso. Résultats d'une enquête sérologique *
}

\author{
Z. Bengaly ${ }^{1}$ R. Ganaba ${ }^{1}$ I. Sidibe ${ }^{1}$ M. Desquesnes ${ }^{1}$
}

\begin{abstract}
Mots-clés
Bovin - Trypanosomose Immunodiagnostic - Epidémiologie Burkina Faso.
\end{abstract}

\begin{abstract}
Résumé
Afin d'estimer l'importance de la trypanosomose bovine dans la zone Sudsoudanienne du Burkina Faso, 1403 bovins ont été sélectionnés par échantillonnage aléatoire dans 18 sites d'élevage répartis dans quatre provinces et soumis à une enquête sérologique pour la détection des anticorps par le test Elisa-indirect. Les prélèvements sanguins ont été effectués entre juillet et septembre 1994 et en mars 1995. La prévalence sérologique sur l'ensemble de la zone étudiée a été évaluée à $43 \pm 2,7$ p. 100. Dans les provinces de la Bougouriba, du Kénédougou et du Mouhoun où l'enquête a été réalisée en saison pluvieuse (juillet à septembre 1994), les prévalences ont été évaluées respectivement à $41,7 \pm 5,6$ p. $100,33,5 \pm 4,4$ p. 100 et $34,3 \pm 5,7$ p. 100 . Dans la province de la Comoé où l'étude a été menée au cours de la saison sèche (mars 1995), la séroprévalence a été estimée $71 \pm 4,5$ p. 100. II n’y a pas eu de relation significative entre le phénotype de l'animal et la séroprévalence observée. En revanche, la séroprévalence a augmenté significativement avec l'âge des animaux.
\end{abstract}

\section{INTRODUCTION}

Au Burkina Faso, la trypanosomose animale est l'une des principales contraintes sanitaires au développement du bétail (11). Elle est présumée endémique dans toute la partie Sud-soudanienne (située entre les isohyètes 1300 et $1000 \mathrm{~mm}$ ), montrant ainsi l'ampleur du problème (6). Possédant d'énormes potentialités pastorales, elle est utilisée comme site d'accueil des troupeaux transhumants. Dans cette zone, des périmètres pastoraux ont été aména-

1. Centre international de recherche-développement sur l'élevage en zone subhumide, 01 BP 454, Bobo-Dioulasso 01, Burkina Faso Fax : (226) 97-23-20 ; e-mail : zakaria_bengaly@hotmail.com

* La première partie de cette étude a paru dans la Revue d'élevage et de médecine vétérinaire des pays tropicaux, 1998, vol. 51, n 3, p. 225-229. gés pour faciliter cette transhumance et des enquêtes épidémiologiques ont été réalisées $(1,2,3)$ pour orienter les stratégies de contrôle des glossines et de la trypanosomose.

Ces études épidémiologiques ont donc été ciblées sur quelques zones prioritaires de développement mais n'ont pas été poursuivies sur l'ensemble de la zone Sud-soudanienne. Afin de compléter celles-ci, une enquête transversale a été réalisée pour évaluer la prévalence des infections et quelques facteurs de sa variation (4). Cette enquête épidémiologique, comme les précédentes, a permis tout au plus d'estimer la prévalence apparente des infections trypanosomiennes, car elle était basée sur l'examen microscopique du buffy coat (12) dont la sensibilité est faible (13). En l'absence d'outils de diagnostic fiables qui permettent une évaluation de la prévalence réelle des infections, la présente étude complète ces investigations par des analyses sérologiques afin d'estimer l'importance de la pression parasitaire due aux trypanosomes. 


\section{MATERIEL ET METHODES}

La zone de l'étude était située dans le sud du Burkina Faso, entre 5 et $12,5^{\circ}$ de lat. $\mathrm{N}$ et 0,5 et $5,5^{\circ}$ de long. $\mathrm{O}$, soit $32 \mathrm{p} .100$ du territoire. Elle était composée de huit provinces avec un effectif bovin estimé à 1205000 têtes, soit 26,6 p. 100 du total. L'étude a porté sur un échantillon de 1403 bovins sélectionnés de façon aléatoire dans quatre provinces. Cet échantillon était composé de trois phénotypes (taurin, zébu, taurin $\mathrm{x}$ zébu) et a été subdivisé en trois classes d'âge ( $<1$ an, 1-2 ans, $>2$ ans) (tableau I).

Les prélèvements sanguins ont été réalisés au niveau de la veine jugulaire à l'aide d'un tube vacutainer hépariné et d'un tube sec. Le sang hépariné a servi à mesurer l'hématocrite et à rechercher les parasites par examen microscopique du buffy coat (13). Le sang non hépariné a été soumis, après 12-24 h de conservation au froid, à une centrifugation différentielle à 3000 tours/min durant $10 \mathrm{~min}$. Le sérum a ensuite été collecté et reparti dans deux cryotubes qui ont été transportés sous froid $\left(4^{\circ} \mathrm{C}\right)$ au laboratoire pour être conservés à $-20{ }^{\circ} \mathrm{C}$ jusqu'à la réalisation des analyses. Les prélèvements sanguins ont été effectués en saison des pluies (juillet à septembre 1994) dans trois des quatre provinces (Bougouriba, Mouhoun, Kénédougou). La quatrième province (Comoé) a été prospectée en saison sèche (mars 1995) pour des raisons d'inaccessibilité en saison des pluies.

Pour la détection des anticorps, les échantillons de sérum ont été analysés par la technique Elisa-indirecte dérivée de celle décrite par Luckins (10). L'antigène utilisé a été la fraction soluble d'un lysat de trypanosomes appartenant au stock Trypanosoma congolense Samandéni 82/CRTA/28 (14). Ce stock a été cultivé chez des souris NMRI irradiées, puis les parasites ont été isolés du sang par chromatographie (9) et lysés par sept cycles de congélation $\left(\right.$ à $-80^{\circ} \mathrm{C}$ ) et de décongélation $\left(\right.$ à $\left.4{ }^{\circ} \mathrm{C}\right)$. La concentration en protéines totales de la fraction soluble a été déterminée par la mesure de l'absorbance à l'aide d'un spectrophotomètre et l'estimation a été faite par le nomograph d'Adams.

L'antigène a été adsorbé dans les plaques de microtitration (Dynatech ; Immulon 1 ; M129A) en déposant dans chaque puits $100 \mu \mathrm{l}$ d'une solution de $\mathrm{Pbs}, \mathrm{pH} \mathrm{7,2} \mathrm{contenant} 0,5 \mu \mathrm{g}$ de protéines, puis en incubant celles-ci pendant $2 \mathrm{~h}$ à $37^{\circ} \mathrm{C}$. Un rinçage des plaques a été réalisé avec une solution de $\mathrm{Pbs}$ contenant $0,1 \mathrm{p} .100$ Tween 20 (Pbst), suivi par une étape de blocage des sites libres avec une solution Pbst contenant 0,2 p. 100 de caséine, en incubation à $37{ }^{\circ} \mathrm{C}$ pendant $1 \mathrm{~h}$. Après un nouveau rinçage, $100 \mu \mathrm{l}$ de sérum dilué 1:200 avec le Pbst contenant 2 p. 100 de lait écrémé (Protifar) ont été déposés dans chaque puits pour une incubation à $37^{\circ} \mathrm{C}$ pendant $30 \mathrm{~min}$. Trois lavages ont été effectués, suivis par le dépôt de $100 \mu \mathrm{l}$ par puits du conjugué anti-IgG bovin (ref. A 5295, Sigma) dilué 1:5 000 avec le Pbst et l'incubation à $37^{\circ} \mathrm{C}$ pendant $30 \mathrm{~min}$. Un nouveau lavage a été réalisé comme précédemment et $100 \mu \mathrm{l}$ par puits du substrat de la réaction colorée (5) ont été déposés dans les plaques pour une incubation de $10 \mathrm{~min}$. Les réactions ont ensuite été stoppées avec une solution d'acide sulfurique et la lecture des absorbances a été réalisée à l'aide d'un spectrophotomètre (Multiskan, MCC 340) utilisé à une longueur d'onde de $405 \mathrm{~nm}$. Les densités optiques obtenues ont été exprimées en pourcentage de positivité par rapport au sérum de référence positif (16). Le seuil de positivité a été évalué à 20 p. 100 en doublant la moyenne des pourcentages de positivité de 200 échantillons de sérum négatifs (collectés à Dori, zone indemne de glossine au nord du Burkina Faso).

La séroprévalence globale a été calculée ainsi que les séroprévalences en fonction des provinces, de la classe d'âge et du phénotype animal. Les comparaisons statistiques, pour analyser l'influence de chacun de ces facteurs sur la séroprévalence, ont été effectuées par la méthode de correction de continuité des intervalles (15). Le test $t$ de Student a été utilisé pour analyser la différence entre les moyennes des hématocrites des animaux séropositifs et séronégatifs.

\section{RESULTATS ET DISCUSSION}

Sur les 1403 bovins qui ont été examinés sur l'ensemble de la zone Sud-soudanienne, 603 ont été détectés séropositifs, soit 43 p. 100 (intervalle de confiance : 40,3-45,6 p. 100). Cette valeur a été 4,8 fois supérieure à la prévalence parasitaire obtenue lors de l'enquête parasitologique (4). La séroprévalence élevée illustre l'importance des contacts entre les animaux et les trypanosomes dans cette zone, mais la présence d'anticorps ne traduit pas l'existence d'une infection courante. L'absence de différence significative entre l'hématocrite moyen des animaux séropositifs $(31,4 \pm 5,9$ p. 100$)$ et celui des séronégatifs $(32,4 \pm 6,1$ p. 100$)$ indique que les anticorps présents proviendraient pour l'essentiel d'infections passées.

La séroprévalence enregistrée en saison pluvieuse a été de 41,7 p. 100 (36,4-47,3 p. 100) dans la Bougouriba, de 33,5 p. 100 (29,4-37,9 p. 100) dans le Kénédougou et de 34,3 p. 100 (29-40 p. 100) dans le Mouhoun. Les différences observées entre ces valeurs n'étaient pas significatives. Cela indique que l'importance des interfaces hôte-parasite a été similaire dans les trois provinces. En revanche, les séroprévalences enregistrées dans ces

\section{Tableau I}

Répartition des animaux échantillonnés en fonction de la province, du phénotype animal et de la classe d'âge

\section{Province}

Taurin

Bougouriba

Comoé

Kénédougou

Mouhoun

Total

* Non déterminé

\section{Phénotype animal}

223

76

311

70

680

99

Zébu Taurin x zébu

92

135

167

230

624

92
5
67
0

20

\begin{tabular}{|c|c|c|c|}
\hline \multicolumn{4}{|c|}{ Classe d'âge } \\
\hline$<1$ an & 1-2 ans & $>2$ ans & Nd * \\
\hline 45 & 101 & 182 & 1 \\
\hline 18 & 58 & 182 & 6 \\
\hline 38 & 105 & 325 & 40 \\
\hline 7 & 49 & 246 & - \\
\hline 108 & 313 & 935 & 47 \\
\hline
\end{tabular}


trois provinces ont été significativement inférieures $(\mathrm{p}<0,05)$ à celle obtenue dans la province de la Comoé où l'enquête a été menée en saison sèche $(71 \pm 4,5$ p. 100). Cette séroprévalence élevée résulte d'une pression glossinienne plus importante pendant la saison sèche du fait de la focalisation des glossines et des bovins autour des points d'eau pérennes (7). En effet, pendant cette période, la répartition des glossines est essentiellement limitée aux formations arborées du réseau hydrographique qui demeurent les seuls points d'abreuvement pour les troupeaux. Ce phénomène n'est pas observé pendant la saison pluvieuse car les glossines sont dispersées dans les galeries forestières et il y a une diversification importante des sources d'approvisionnement en eau. Par ailleurs, la plupart des animaux dans cette zone sont traditionnellement traités avec les trypanocides en début de saison pluvieuse (mai et juin) ce qui peut favoriser leur séroconversion négative.

L'analyse de la séroprévalence en fonction du phénotype animal indique des valeurs de 48,4 p. $100(38,3-58,6$ p. 100) chez les taurins, de 44,1 p. $100(40,3-47,9$ p. 100) chez les zébus et de 40,8 p. 100 (36,9-44,9 p. 100) chez les métis (Baoulé x zébu). Les différences observées ne sont pas significatives, ce qui suggère que les trois phénotypes ont été soumis à une pression parasitaire identique. En revanche, la séroprévalence chez les bovins de plus de 2 ans $(47,1$ p. $100 ; 45,1-51,6$ p. 100) a été significativement supérieure $(\mathrm{p}<0,05)$ à celle des animaux de 1 à 2 ans $(30,6$ p. 100 ; 25,6-36,1 p. 100) et cette dernière a été significativement supérieure $(\mathrm{p}<0,05)$ à celle obtenue chez les animaux de moins de 1 an (16,6 p. $100 ; 10,4-25,3$ p. 100). Dans les trois classes d'âge, aucune différence significative n'a été obtenue entre les Baoulés, les zébus et les métis. L'ensemble de ces observations est conforme aux résultats obtenus précédemment par Desquesnes et coll. (8) dans le secteur de Sidéradougou.
L'augmentation de la séroprévalence avec l'âge pourrait résulter du mode d'élevage car les veaux sont généralement à l'attache à côté du parc et ne vont pas au pâturage, ce qui limite les possibilités de contact avec les glossines. Les sérologies positives chez les veaux résultent, donc, soit d'anticorps maternels, soit d'infections actives, mais leur importance relative ne peut être évaluée que dans le cadre d'un suivi épidémiologique individuel. Ils sont, en revanche, progressivement intégrés au fur et à mesure qu'ils prennent de l'âge, dans le troupeau adulte qui va, dans sa pâture, au contact des glossines et du parasite.

\section{- CONCLUSION}

La présente étude a permis de déterminer l'importance de la pression parasitaire et d'analyser l'effet du phénotype et de l'âge des animaux. Il en résulte une meilleure connaissance de la trypanosomose bovine dans la zone Sud-soudanienne du Burkina Faso. Les informations générées devraient contribuer à une meilleure orientation des stratégies de contrôle de la trypanosomose dans la zone étudiée.

Toutefois, des enquêtes épidémiologiques longitudinales dans les zones prioritaires de développement pastoral demeurent la meilleure approche pour une gestion rationnelle du problème de la trypanosomose bovine.

\section{Remerciements}

Ce projet a été financé par le Cirad-emvt (programme Santé animale), le Geprenaf et l'Aiea (Animal Health and Production Section). Les auteurs expriment leur profonde gratitude au Professeur Gérard Duvallet, Université de Montpellier III, France, qui a initié ce travail avant de quitter le Cirdes en 1994.

\section{BIBLIOGRAPHIE}

1. BAUER B., AMSLER-DELAFOSSE S., CLAUSEN P.H., KABORE I., PETRICH-BAUER J., 1995. Successful application of deltamethrin pouron to cattle in a campaign against tsetse flies (Glossina spp.) in the pastoral zone of Samorogouan. Trop. Med. Parasitol., 46: 183-189.

2. BAUER B., KABORE I., LIEBISCH A., MEYER F., PETRICH-BAUER J., 1992. Simultaneous control of ticks and tsetse flies in Satiri, Burkina Faso, by the use of flumethrin pour-on for cattle. Trop. Med. Parasitol., 43: 41-46.

3. BAUER B., PETRICH-BAUER J., KABORE I., KOUROUMA B., MATTAUSCH M., SOME J., TAMBOURA I., 1988. Epidemiological survey in the pastoral zone of Sideradougou, Burkina Faso. In: Proc. Int. Symp. Modern Insect Control, Vienna, Austria, 16-20 November 1987. Vienna, Austria, IAEA, p. 139-149.

4. BENGALY Z., GANABA R., SIDIBE I., DUVALLET G., 1998. Infections trypanosomiennes chez des bovins dans la zone Sud-soudanienne du Burkina Faso. Revue Elev. Méd. vét. Pays trop. 51 : 225-229.

5. BOCQUENTIN R., DUVALLET G., 1990. Amélioration de la reproductibilité du test Elisa adapté à la détection d'anticorps antiTrypanosoma congolense chez les bovins. Revue Elev. Méd. vét. Pays trop., 43 : 179-186.

6. CHALLIER A., LAVEISSIERE C., 1977. La répartition des glossines en Haute-Volta. Une carte couleur au $1 / 200000^{\mathrm{e}}$ et notice explicative. Paris, France, Orstom.

7. DE LA ROCQUE S., BENGALY Z., MICHEL J.F., SOLANO P., SIDIBE I., CUISANCE D., 1999. Importance des interfaces spatiales et temporelles entre les bovins et les glossines dans la transmission de la trypanosomose animale en Afrique de l'Ouest. Revue Elev. Méd. vét. Pays trop., 52 : 215-222.

8. DESQUESNES M., MICHEL J.F., DE LA ROCQUE S., SOLANO P., MILLOGO L., BENGALY Z., SIDIBE I., 1999. Enquête parasitologique et sérologique (Elisa-indirect) sur les trypanosomoses des bovins dans la zone de Sidéradougou, Burkina Faso. Revue Elev. Méd. vét. Pays trop., 52 : 223-232

9. LANHAM S.M., GODFREY D.G., 1970. Isolation of salivarian trypanosomes from Man and other mammals using DEAE-Cellulose. Exp. Parasitol., 28: 521-534.

10. LUCKINS A.G., 1977. Detection of antibodies in trypanosomeinfected cattle by means of a microplate enzyme-linked immunosorbent assay. Trop. Anim. Health Prod., 9: 53-62.

11. MARA, 1989. Etude prospective du sous-secteur élevage au Burkina Faso. Rapport de synthèse, tome I. Paris, France, ministère de I'Agriculture et des ressources animale, $364 \mathrm{p}$.

12. MURRAY M., MURRAY P.K., MCINTYRRE W.I.M., 1977. An improved parasitological technique for the diagnosis of African trypanosomiasis. Trans. R. Soc. trop. Med. Hyg., 1: 325-326.

13. PARIS J., MURRAY M., MCODIMBA F.A., 1982. A comparative evaluation of the parasitological techniques currently available for the diagnosis of African trypanosomosis in cattle. Acta trop., 39: 307-316.

14. ROELANTS G.E., PINDER M., 1987. The virulence of Trypanosoma congolense can be determined by the antibody response of inbred strains of mice. Parasite Immunol., 9: 379-388.

15. VOLLSET S.E., 1993. Confidence interval for the binomial proportion. Stat. Med., 12: 809-824.

16. WRIGHT P.F., NILSON E., VAN ROOIJ E.M.A., LELANTA M., JEGGO M.H., 1993. Standardisation and validation of enzyme-linked immunosorbent assay techniques for the detection of antibody in infectious disease diagnosis. Revue sci. tech. Off. int. Epizoot., 12: 435-450.

Accepté le 14.06.2002 


\section{Summary}

Bengaly Z., Ganaba R., Sidibe I., Desquesnes M. Bovine Trypanosomosis in the South-Sudan Zone of Burkina Faso. Results of a Serological Survey

A serological survey was performed to assess the importance of bovine trypanosomosis in the South-Sudan zone of Burkina Faso. In total, 1403 head of cattle were randomly examined by indirect-ELISA for the detection of antibodies in 18 farming sites spread out in four provinces. Blood sampling was carried out between July and September 1994, and in March 1995. The overall seroprevalence was estimated at $43 \pm 2.6 \%$. The seroprevalence was estimated at $41.7 \pm 5.6 \%, 33.5 \pm 4.4 \%$ and $34.3 \pm 5.7 \%$ in the provinces of Bougouriba, Kenedougou and Mouhoun, respectively, during the rainy season (July-September 1994), whereas it was estimated at $62.5 \pm 4.6 \%$ in the province of Comoe during the dry season (March 1995). No significant relationship was observed between the animal phenotype and the seroprevalence. In contrast, the seroprevalence increased significantly with the animal age.

Key words: Cattle - Trypanosomosis - Immunodiagnosis Epidemiology - Burkina Faso.

\section{Resumen}

Bengaly Z., Ganaba R., Sidibe I., Desquesnes M. Tripanosomosis bovina en la zona Sur sudanesa de Burkina Faso. Resultados de una encuesta serológica

Con el fin de estimar la importancia de la tripanosomosis bovina en la zona Sur sudanesa de Burkina Faso, se seleccionaron 1403 bovinos, mediante muestreos aleatorios en 18 sitios de cría repartidos en cuatro provincias y fueron tomadas muestras para la detección de anticuerpos por ELISA indirecto. Las muestras sanguíneas fueron efectuadas entre julio y septiembre de 1994 y en marzo de 1995. La prevalencia serológica sobre el conjunto de la zona estudiada se evaluó a $43 \pm 2,6 \%$. En las provincias de Bougouriba, de Kenedougou y de Mouhoun, en donde la encuesta fue realizada durante la estación lluviosa (de julio a septiembre de 1994), las prevalencias fueron evaluadas respectivamente a 41,7 \pm $5,6 \%, 33,5 \pm 4,4 \%$ y $34,3 \pm 5,7 \%$. En la provincia de Comoe, en donde el estudio se realizó durante la estación seca (marzo de 1995), la prevalencia sérica fue estimada a $62,5 \pm 4,6 \%$. No se encontró relación significativa entre el fenotipo de los animales y la prevalencia sérica. Por otro lado, la prevalencia sérica aumentó significativamente con la edad de los animales.

Palabras clave: Ganado bovino - Tripanosomosis - Inmunodiagnosis - Epidemiología - Burkina Faso. 